\title{
Brain-targeting study of stearic acid-grafted chitosan micelle drug-delivery system
}

This article was published in the following Dove Press journal:

International Journal of Nanomedicine

28 June 2012

Number of times this article has been viewed

\section{Yi-Ting Xie \\ Yong-Zhong Du \\ Hong Yuan \\ Fu-Qiang $\mathrm{Hu}$}

College of Pharmaceutical Sciences, Zhejiang University, Hangzhou, China
Purpose: Therapy for central nervous system disease is mainly restricted by the blood-brain barrier. A drug-delivery system is an effective approach to overcome this barrier. In this research, the potential of polymeric micelles for brain-targeting drug delivery was studied.

Methods: Stearic acid-grafted chitosan (CS-SA) was synthesized by hydrophobic modification of chitosan with stearic acid. The physicochemical characteristics of CS-SA micelles were investigated. bEnd.3 cells were chosen as model cells to evaluate the internalization ability and cytotoxicity of CS-SA micelles in vitro. Doxorubicin (DOX), as a model drug, was physically encapsulated in CS-SA micelles. The in vivo brain-targeting ability of CS-SA micelles was qualitatively and quantitatively studied by in vivo imaging and high-performance liquid chromatography analysis, respectively. The therapeutic effect of DOX-loaded micelles in vitro was performed on glioma C6 cells.

Results: The critical micelle concentration of CS-SA micelles with $26.9 \% \pm 1.08 \%$ amino substitute degree was $65 \mu \mathrm{g} / \mathrm{mL}$. The diameter and surface potential of synthesized CS-SA micelles in aqueous solution was $22 \pm 0.98 \mathrm{~nm}$ and $36.4 \pm 0.71 \mathrm{mV}$, respectively. CS-SA micelles presented excellent cellular uptake ability on bEnd.3 cells, the $\mathrm{IC}_{50}$ of which was $237.6 \pm 6.61 \mu \mathrm{g} / \mathrm{mL}$. DOX-loaded micelles exhibited slow drug-release behavior, with a cumulative release up to $72 \%$ within 48 hours in vitro. The cytotoxicity of DOX-loaded CS-SA micelles against C6 was $2.664 \pm 0.036 \mu \mathrm{g} / \mathrm{mL}$, compared with $0.181 \pm 0.066 \mu \mathrm{g} / \mathrm{mL}$ of DOX $\cdot \mathrm{HCl}$. In vivo imaging results indicated that CS-SA was able to transport rapidly across the blood-brain barrier and into the brain. A maximum DOX distribution in brain of 1.01\%/g was observed 15 minutes after administration and maintained above $0.45 \% / g$ within 1 hour. Meanwhile, free $\mathrm{DOX} \cdot \mathrm{HCl}$ was not detected in brain. In other major tissues, DOX-loaded micelles were mainly distributed into lung, liver, and spleen, with a reduction of DOX accumulation in heart.

Conclusion: The CS-SA micelles were able to be used as a promising carrier for a braintargeting drug delivery system.

Keywords: chitosan, stearic acid, micelle, blood-brain barrier, brain targeting, in vivo imaging

\section{Introduction}

There has been an increasing population worldwide suffering various central nervous system disorders, such as Alzheimer's disease, Parkinson's, cerebral ischemia, and brain tumor. The treatment for these diseases is still confronting a big challenge due to the existence of the blood-brain barrier (BBB), which prevents most drug molecules from entering the brain. The BBB controls substance flow in and out of the brain with precision and strictness, so as to maintain the internal environment of the brain. Besides the physical barrier characterized by the tight junction, the brain capillary
Correspondence: Fu-Qiang Hu

College of Pharmaceutical Sciences,

Zhejiang University, 866 Yuhangtang

Road, Hangzhou 310058, China

Tel $+8657 \mid 8820844$ I

Fax+86 57। 88208439

Email hufq@zju.edu.cn 
endothelial cells highly express the active efflux system. ${ }^{1}$ The system that is representative of P-glycoprotein (member of adenosine triphosphate-binding cassette P-gp) greatly reinforces BBB function by effectively removing drugs from the brain and pumping them back into blood. ${ }^{2}$ Therefore, the development of brain-targeting drug-delivery systems has become an urgent matter.

A number of attempts have been made to overcome the barrier. Various nanoparticle delivery systems were designed for brain targeting, including liposomes, solid lipid nanoparticles, and polymeric micelles. Among these, polymeric micelles have emerged as an ideal candidate for brain-targeting delivery. The amphiphilic copolymer can self-assemble into micelles in aqueous solution with a core-shell structure. The hydrophobic core acts as the drug-loading depot, with the surrounding hydrophilic block to increase stabilization. Incorporation of low molecules into micelles can effectively enhance drug solubility and improve drug pharmacokinetics and biodistribution. ${ }^{3}$ Polymeric micelles possess many advantageous properties: small particle size that allows escaping from the reticuloendothelial system, stability in plasma, and flexibility of modification with targeting ligands. Compared to liposomes, polymeric micelles require less excipients and are easier to formulate, with high physical stability and sustained drug release. ${ }^{4}$ Various biodegradable polymers such as poly(lactic acid), poly(lactic-co-glycolic acid), poly(butylcyanoacrylate), chitosan (CS), and polylysine have been exploited as brain-targeting drug-delivery systems. ${ }^{5-7}$ These carrier systems have successfully delivered poorly diffused drugs into the brain, including doxorubicin (DOX), loperamide, and dalargin. ${ }^{8-12}$

It is widely accepted that free DOX is not able to transport across the $\mathrm{BBB}$, which is mainly associated with the active efflux system on the BBB. ${ }^{13-15}$ Known as a biodegradable and biocompatible material, $\mathrm{CS}$ with its low molecular weight is studied widely as a drug carrier due to its good solubility in physiological conditions. ${ }^{16}$ Studies have shown that CS can transiently open tight junctions, resulting in a paracellular pathway of material through the epithelial barrier. ${ }^{17}$ In our previous studies, stearic acid-grafted CS (CS-SA) was synthesized using low molecular-weight CS, which was able to form micelle-like nanoparticles in the aqueous phase, and has been used to deliver gene ${ }^{18}$ and antitumor agents like doxorubicin ${ }^{19,20}$ and paclitaxel. ${ }^{21}$ All the results demonstrated the excellent biomembrane penetration ability of CS-SA, indicated by rapid in vitro cellular uptake and comparable in vivo therapeutic effect with a commercial agent. Moreover, the micelles were able to reserve multidrug resistance by avoiding recognition by P-gp. The ability of CS-SA to open transiently biobarriers and bypass the P-gp system by application on oral drug delivery was further confirmed. ${ }^{22}$ The purpose of this study was to explore the potential of CS-SA micelles as a brain-targeting delivery system. Stearic acid (SA) was conjugated onto CS with 18 -KDa weight average molecular weight using 1-ethyl-3-(3-dimethylaminopropyl) carbodiimide (EDC)as the coupling reagent. ${ }^{18,19}$ The physicochemical characteristics of CS-SA were then investigated. In vitro cellular uptake and cytotoxicity were performed on bEnd. 3 cells. In vivo imaging was applied to track the route of micelles after administration. DOX was chosen as the model drug and encapsulated into CS-SA micelles to prepare DOX-loaded micelles. The in vivo distribution of DOX was conducted in a quantitative way.

\section{Materials and methods Materials}

CS with an average molecular weight of $18 \mathrm{kDa}$ was obtained by enzymatic degradation from CS $(\mathrm{Mw}=450.0 \mathrm{kDa}, 95 \%$ deacetylated degree; Yuhuan Marine Biochemistry, Zhejiang, China). SA was purchased from Chemical Reagent (Shanghai, China). EDC, 2,4,6-trinitrobenzene sulfonic acid (TNBS), and 3-(4, 5-dimethylthiazol-2-yl)-2,5-diphenyl-tetrazolium bromide (MTT) came from Sigma (St Louis, MO). DiR dye was a product of Molecular Probes (Eugene, OR). Dulbecco's modified Eagle's medium (DMEM), fetal bovine serum (FBS), and trypsin-ethylenediaminetetraacetic acid (EDTA) were purchased from Gibco (Gaithersberg, MD). DOX hydrochloride DOX $\cdot \mathrm{HCl}$ was gifted by Zhejiang Hisun Pharmaceutical (Taizhou, China). All other chemicals were of analytical or chromatographic grade.

ICR mice and Sprague Dawley (SD) rats (180-200g, male) were obtained from Experimental Animal Center of Zhejiang University and maintained at $22 \pm 2^{\circ} \mathrm{C}$ on a 12-hour light-dark cycle with access to food and water. The animals used for the experiment were treated according to the protocols evaluated and approved by the ethical committee of Zhejiang University.

\section{Synthesis of CS-SA copolymer}

The CS-SA conjugate was synthesized via the reaction of carboxyl groups of stearic acid (SA) with amine groups of $\mathrm{CS}$ in the presence of EDC. ${ }^{23}$ It mainly followed two steps. Firstly, SA $(0.7 \mathrm{~g})$ with EDC (SA:EDC $=1: 10, \mathrm{~mol}: \mathrm{mol})$ were dissolved in $80 \mathrm{~mL}$ of ethanol and reacted at $60^{\circ} \mathrm{C}$ by stirring in a water bath for 30 minutes. Secondly, $1 \mathrm{~g}$ of CS was dissolved in $120 \mathrm{~mL}$ of pure water and incubated to $80^{\circ} \mathrm{C}$. The organic mixture was added into the CS solution 
under intensive stirring and kept reacting for 6 hours. The resultant mixture was dialyzed against deionized (DI) water for 48 hours using a dialysis membrane (MWCO $7 \mathrm{kDa}$; Spectrum Laboratories, Laguna Hills, CA) followed by lyophilization. The lyophilized product was further washed with ethanol to remove the unreacted SA. Finally, the product CS-SA was redispersed in DI water and lyophilized.

\section{Characterization of CS-SA}

The ${ }^{1} \mathrm{H}$ NMR spectra of CS-SA were obtained by a nuclear magnetic resonance spectrometer (AC-80; Bruker BioSpin, Rheinstetten, Germany). CS-SA was dissolved in $\mathrm{D}_{2} \mathrm{O}$ at a concentration of $20 \mathrm{mg} / \mathrm{mL}$ before testing.

The critical micelle concentration of CS-SA in aqueous medium was estimated by spectroscopy using pyrene as a probe. ${ }^{18}$ The fluorescence emission spectrum of pyrene was obtained by a fluorometer (F-2500; Hitachi, Tokyo, Japan). The excitation wavelength was $337 \mathrm{~nm}$, and the slits were set at $2.5 \mathrm{~nm}$ (excitation) and $10 \mathrm{~nm}$ (emission), respectively. The concentrations of CS-SA solution with $5.93 \times 10^{-7} \mathrm{M}$ pyrene were varied from $5.0 \times 10^{-3}$ to $1.0 \mathrm{mg} / \mathrm{mL}$. The intensity ratio of the first peak $\left(\mathrm{I}_{1}, 374 \mathrm{~nm}\right)$ to the third peak $\left(\mathrm{I}_{3}, 385 \mathrm{~nm}\right)$ in the emission spectra of pyrene was calculated.

The degrees of amino substitution of CS-SA were determined by 2,4,6-trinitrobenzene sulfonic acid (TNBS) method. ${ }^{19}$ Briefly, $3.0 \mathrm{~mL}$ of CS-SA solution at a concentration of $0.1 \mathrm{mg} / \mathrm{mL}$ was incubated with $2.0 \mathrm{~mL}$ of $4 \%$ $\mathrm{NaHCO}_{3}$ and $2.0 \mathrm{~mL}$ of $0.1 \%$ TNBS at $37^{\circ} \mathrm{C}$ for 2 hours. Following addition of $2.0 \mathrm{~mL}$ of $\mathrm{HCl}(2.0 \mathrm{~mol} / \mathrm{L})$, the mixture was measured at $344 \mathrm{~nm}$ by a ultraviolet spectrophotometer (TU-1800PC; Beijing Purkinje General Instrument, Beijing, China).

The hydrodynamic diameter and zeta potential of the micelles of CS-SA $(1.0 \mathrm{mg} / \mathrm{mL})$ were measured by dynamic light scattering using a Zetasizer (3000 HS; Malvern Instruments, Malvern, UK). Each sample determination was done in triplicate.

The morphological observation of CS-SA micelles was performed by transmission electron microscopy (TEM) (JEM-1230; Jeol, Tokyo, Japan). The samples were stained with $2 \%(\mathrm{w} / \mathrm{v})$ phosphotungstic acid and placed on copper grids with films for viewing by TEM.

\section{Preparation of DOX-loaded CS-SA micelles}

DOX was obtained by the alkalization of $\mathrm{DOX} \cdot \mathrm{HCl}$ with twice the molar numbers of triethylamine in dimethyl sulfoxide (DMSO) overnight and used for the preparation of
DOX-loaded CS-SA (CS-SA/DOX) micelles. ${ }^{24}$ To prepare CS-SA/DOX micelles, $200 \mathrm{mg}$ of CS-SA was dissolved in $100 \mathrm{~mL}$ of DI water, followed by the addition of $30 \mathrm{~mL}$ of DOX-DMSO solution $(1.0 \mathrm{mg} / \mathrm{mL})$ in drops. The mixture was dialyzed against DI water using a dialysis membrane (MWCO $7 \mathrm{kDa}$ ) for 24 hours. It was centrifuged at $4000 \mathrm{rpm}$ for 10 minutes to remove unentrapped drug, and the final product was obtained by lyophilization.

\section{Characterization of DOX-loaded CS-SA micelles}

Micellar size and zeta potential of CS-SA/DOX micelles with the CS-SA concentration of $1.0 \mathrm{mg} / \mathrm{mL}$ were performed using the Zetasizer analyzer. All measurements were performed in triplicate.

The DOX content encapsulated in CS-SA micelles was measured by the F-2500 spectrophotometer. The excitation wavelength and emission wavelength were set at $505 \mathrm{~nm}$ and $565 \mathrm{~nm}$, respectively, both with slit openings of $5 \mathrm{~nm}$. The drug encapsulation efficiency (EE) and drug loading capacity (DL) of CS-SA/DOX were estimated by centrifugal ultrafiltration method. Briefly, a part of CS-SA/DOX micelle solution was centrifuged in ultrafiltration tubes (Microcon YM-10, MWCO 3000 kDa; Millipore, Billerica, MA). The DOX concentration $(\mathrm{C}, \mu \mathrm{g} / \mathrm{mL})$ of ultrafiltrate was determined to evaluate the amount of the free drug. Another part of CS-SA/DOX solution was diluted 100-fold by DMSO aqueous solution (DMSO: $\mathrm{H}_{2} \mathrm{O}=9: 1, \mathrm{v}: \mathrm{v}$ ), the DOX concentration $\left(\mathrm{C}_{0}, \mu \mathrm{g} / \mathrm{ml}\right)$ of which was considered as the total drug amount. The EE (\%) and DL (\%) of CS-SA/DOX was calculated by the following equations:

$$
\begin{gathered}
\mathrm{EE} \%=\left(\mathrm{C}_{0} \times 100-\mathrm{C}\right) \times \mathrm{V} / \mathrm{Ma} \times 100 \% \\
\mathrm{DL} \%=\left(\mathrm{C}_{0} \times 100-\mathrm{C}\right) \times \mathrm{V} /\left(1000+\left[\mathrm{C}_{0} \times 100-\mathrm{C}\right]\right. \\
\times \mathrm{V}) \times 100 \%
\end{gathered}
$$

where $\mathrm{Ma}$ is the charged amount of drug, $\mathrm{mg}$; and $\mathrm{V}$ is the total volume of CS-SA/DOX solution, $\mathrm{mL}$.

\section{In vitro release study}

In vitro release studies of DOX from CS-SA micelles were performed in phosphate-buffered saline (PBS) ( $\mathrm{pH}$ 7.2). The saturated solubility of DOX in PBS ( $\mathrm{pH} 7.2)$ was measured to be $76 \mu \mathrm{g} / \mathrm{mL}$. Briefly, $1.0 \mathrm{~mL}$ of CS-SA/DOX micelle solution (containing $75 \mu \mathrm{g}$ of DOX) was added into the dialysis filter (MWCO $7 \mathrm{kDa}$ ) and put into a plastic tube containing $20 \mathrm{~mL}$ of PBS ( $\mathrm{pH}$ 7.2) solution. The plastic 
tube was then placed in an incubator shaker (HZ-8812S; Scientific and Educational Equipment, Taicang, China), which was maintained at $37^{\circ} \mathrm{C}$ and shaken horizontally at $65 \mathrm{rpm}$. At predetermined time intervals, sample solution was withdrawn and release medium was totally replaced with fresh PBS solution. The drug concentration was determined by fluorescence spectrophotometer. All drug-release tests were performed in triplicate.

\section{Cell culture}

bEnd. 3 cells (immortalized mouse brain endothelial cell line) and murine C6 glioma cells were donated by the Pharmacolgy Department, College of Medicine, Zhejiang University (Hangzhou, China). For both bEnd.3 and C6, cells were maintained in DMEM supplemented with $10 \%$ (v/v) FBS and penicillin/streptomycin $(100 \mathrm{U} / \mathrm{mL}, 100 \mathrm{U} / \mathrm{mL})$ at $37^{\circ} \mathrm{C}$ in a humidified atmosphere containing $5 \% \mathrm{CO}_{2}$. Cells were subcultured regularly using trypsin-EDTA.

\section{In vitro cellular uptake and cytotoxicity}

To visualize cellular uptake of micelles, CS-SA was labeled with fluorescein isothiocyanate (FITC). FITC was conjugated onto CS-SA through the reaction between the amino group of CS-SA and the isothiocyanate of FITC. ${ }^{20}$ Briefly, $10 \mathrm{mg}$ of CS-SA was dissolved in $5.0 \mathrm{~mL}$ of DI water and an aliquot of FITC ethanol solution (CS-SA:FITC = 1:1, mol:mol) was added in drops. The resultant solution was kept stirring overnight at room temperature. The final product was obtained after dialysis against DI water for 24 hours.

bEnd. 3 cells were seeded at $2 \times 10^{4}$ cells/well in a 24-well plate (Nalge Nunc, Naperville, IL) and grown for 24 hours. Aliquots of FITC-CS-SA (containing $100 \mu \mathrm{g}$ of CS-SA) were added, and the cells were further incubated for predetermined time intervals. At the end of the experiment, cells were washed with PBS three times and cellular uptake was observed by fluorescence microscopy (Olympus America, Melville, NY).

bEnd. 3 was used to evaluate the cytotoxicity of CS-SA in vitro by MTT assay. Briefly, $1 \times 10^{4}$ of bEnd. 3 cells were planted in 96-well plates (Nalge Nunc) and grown for 24 hours. The cells were exposed to a series of concentrations of CS-SA micelles at $37^{\circ} \mathrm{C}$ for another 48 hours. At the end of incubation, $20 \mu \mathrm{L}$ of MTT solution with a concentration of $5 \mathrm{mg} / \mathrm{mL}$ was added and incubated for further 4 hours at $37^{\circ} \mathrm{C}$. Each well was then washed with PBS after the medium was removed, and then $100 \mu \mathrm{L}$ of DMSO was added to dissolve MTT formazan crystals. Finally, the plate was shaken for 10 minutes, and the absorbance of formazan product was measured at $570 \mathrm{~nm}$ in a microplate reader (Model 680;
Bio-Rad, Hercules, CA). Cell viability was expressed as percentage of absorbance in comparison with that of the control, which comprised the cells without exposure to the samples. All the experiments were performed in triplicate. The cytotoxicity of CS-SA/DOX micelles against C6 cells was obtained in a similar process, except that $\mathrm{C} 6$ cells were exposed to a series of DOX concentrations. Free DOX $\cdot \mathrm{HCl}$ was set as positive control.

\section{In vivo imaging}

The fluorescent dye DiR was physically entrapped into micelles to keep track of micelles in vivo. In brief, $10 \mathrm{mg}$ of CS-SA was dissolved in $2.0 \mathrm{~mL}$ of DI water, followed by the addition of $20 \mu \mathrm{L}$ of DiR-DMSO solution $(5.0 \mathrm{mg} / \mathrm{mL})$ in drops. The mixture was subjected to ultrasonic treatment in a water bath for 30 minutes, followed by dialysis against DI water using a dialysis membrane (MWCO $7 \mathrm{kDa}$ ) for 24 hours. The DiR-loaded CS-SA micelle solution (CS-SA/ DiR) was finally obtained.

Before the experiment, the animals were anesthetized. Then, the CS-SA/DiR solution was injected via tail vein of nude mice at a dose of $25 \mathrm{mg}$ CS-SA $/ \mathrm{kg}$. Images were taken by Maestro EX in vivo imaging system (CRI, Woburn, MA) at predetermined time points after injection. Brain was extirpated 0.5 hours after injection for fluorescence image. Blank brain served as negative control.

\section{Biodistribution study}

The pharmacokinetics of DOX-loaded micelles was studied in Sprague Dawley rats. Rats were divided into two groups, each group containing five rats. $\mathrm{DOX} \cdot \mathrm{HCl}$ solution and DOX-loaded micelles were administered intravenously, both at a dose of $1 \mathrm{mg} \mathrm{DOX} / \mathrm{kg}$. Blood samples were collected from retro-orbital sinus into heparinized tubes at each time point ( 5 minutes, 15 minutes, 30 minutes, 1 hour, 2 hours, and 4 hours) after administration. The blood was centrifuged at $3000 \mathrm{rpm}$ for 10 minutes, and plasma was obtained for determination of DOX concentration.

Biodistribution study of CS-SA/DOX micelles was carried out in ICR mice in comparison with DOX HCl. CS-SA/DOX micelles and $\mathrm{DOX} \cdot \mathrm{HCl}$ solution were injected via tail vein of ICR mice, both at a dose of $2.5 \mathrm{mg} \mathrm{DOX} / \mathrm{kg}$. At 5 minutes, 15 minutes, 30 minutes, 1 hour, 2 hours, and 4 hours after administration, brains were taken out and rinsed with PBS. Brain samples were carefully handled to remove residual blood and capillaries and dried with filter paper. All samples were weighed and kept at $-80^{\circ} \mathrm{C}$ before analysis. Other major tissues (including heart, liver, spleen, lung, and kidney) 
were used for distribution study at 0.5 and 2 hours after administration. Three mice were used for each time point.

\section{Processing of tissues for HPLC analysis}

Plasma samples were prepared according to the following procedure. In brief, $1.5 \mathrm{~mL}$ of chloroform/methanol $(1: 2, \mathrm{v}: \mathrm{v})$ was added to $0.2 \mathrm{~mL}$ of plasma, followed by vortexing and centrifugation ( $8000 \mathrm{rpm}, 10$ minutes). The supernatant was transferred into a plastic tube and dried at $37^{\circ} \mathrm{C}$ under a stream of $\mathrm{N}_{2}$. The residual was redissolved in methanol by ultrasound treatment in an ice bath for 15 minutes. After centrifugation $\left(15,000 \mathrm{rpm}, 4^{\circ} \mathrm{C}\right.$ and 10 minutes) the supernatant was subjected to high-performance liquid chromatography (HPLC) analysis. In the case of tissue samples, these were homogenized with PBS ( $\mathrm{pH}$ 7.6) in a high-speed tissue homogenizer under an ice bath (JY92; Scientz, Ningbo, China), and $0.4 \mathrm{~mL}$ of the homogenate was subjected to the same procedure as for plasma samples. Blank plasma or homogenates were spiked with appropriate DOX-loaded micelle solutions. Control samples of plasma or tissues for drug analysis were prepared in the same manner.

HPLC analysis for doxorubicin was performed under a modular liquid chromatograph system (Agilent, Santa Clara, CA) with a fluorescence detector. A Diamonsil TM C18 (Dikma Technologies, Beijing, China) column $(250 \mathrm{~mm} \times 4.6 \mathrm{~mm}, 5 \mu \mathrm{m})$ was used. The mobile phase consisted of acetonitrile and water containing $10 \mathrm{mM}$ phosphoric acid and $20 \mathrm{mM}$ lauryl sodium sulfate (80:20, v:v) and the flow rate was kept at $1.0 \mathrm{~mL} / \mathrm{min}$. The column temperature was set to be $30^{\circ} \mathrm{C}$. The effluent was monitored at an excitation of $505 \mathrm{~nm}$ and an emission of $555 \mathrm{~nm}$.

$\mathrm{DOX} \cdot \mathrm{HCl}$ was extracted from tissue samples as previously reported. ${ }^{25}$ Before processing, blood samples were added with same volume of borate buffer solution ( $\mathrm{pH} 9.6$ ) and tissues were homogenized with PBS solution ( $\mathrm{pH}$ 7.6). Tissue homogenate or blood samples was extracted by chloroform/ methanol (4:1, v:v). The extracts were then subjected to HPLC analysis according to the method of Yu et al..$^{25}$

\section{Results}

\section{Synthesis of CS-SA}

The CS-SA was obtained by the reaction between the carboxyl group of SA and the amino group of CS oligosaccharide using EDC as the coupling reagent. The chemical structure of CS-SA was confirmed by ${ }^{1} \mathrm{H}$ NMR spectra (Figure 1). The $0.9 \mathrm{ppm}$ and $1.0 \mathrm{ppm}$ of chemical shift could be respectively attributed to the methyl hydrogen and methylene hydrogen of the stearate group. Therefore, it was

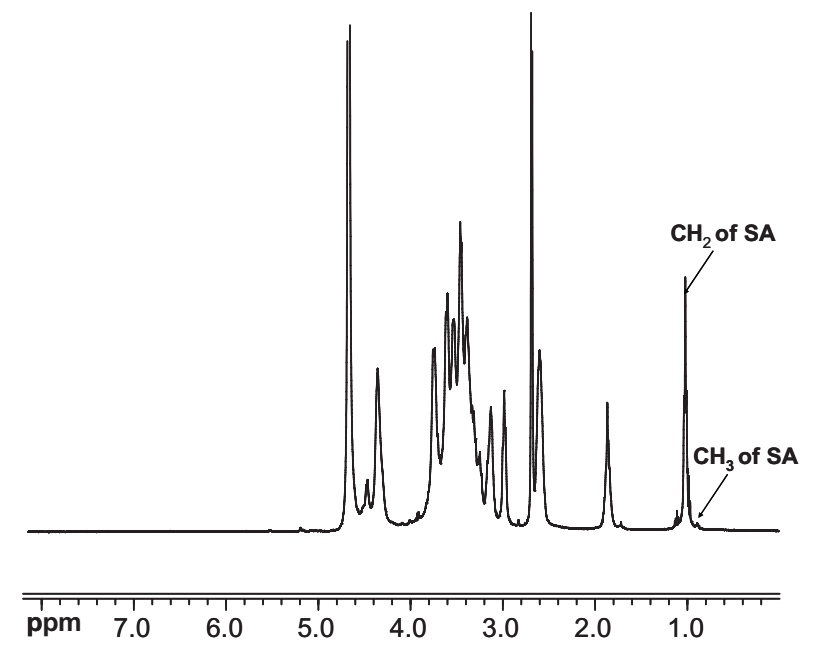

Figure I 'H NMR spectra of stearic acid-grafted chitosan (CS-SA) conjugate.

assumed that the SA had been successfully grafted onto CS. According to the TNBS test, the amino substitute degree of CS-SA was determined to be $26.9 \% \pm 1.08 \%$.

\section{Characterization of CS-SA micelles}

The synthesized CS-SA was able to self-assemble to form micelles in aqueous solution. As seen in Table 1, CS-SA micelles exhibited an average number diameter of $22 \pm 0.98 \mathrm{~nm}$, consistent with the TEM observation results (Figure 2), which showed a regular spherical morphology of CS-SA micelles. The relatively high zeta potential of $36.4 \pm 0.71 \mathrm{mV}$ helped to increase the stability of micelles by repulsion interaction. The concentration of CS-SA plotted against $\mathrm{I}_{1} / \mathrm{I}_{3}$ is presented in Figure 2. The critical micelle concentration of CS-SA measured by fluorescence was $65 \mu \mathrm{g} / \mathrm{mL}$, which indicated that CS-SA micelles had good self-assembling ability.

\section{In vitro cellular uptake and cytotoxicity}

The FITC-CS-SA was synthesized and the cellular uptake was observed using fluorescence microscopy. As shown in Figure 3, distribution of FITC-CS-SA micelles in cells was observed after 0.5 hour's incubation. Fluorescence

Table I Characteristics of CS-SA micelles and DOX loaded CS-SA micelles

\begin{tabular}{lrllll}
\hline Micelles & \multicolumn{1}{l}{$\begin{array}{l}\boldsymbol{d}_{\mathbf{n}} \\
(\mathbf{n m})\end{array}$} & PI & $\begin{array}{l}\zeta \\
(\mathbf{m V})\end{array}$ & $\begin{array}{l}\text { EE } \\
(\%)\end{array}$ & $\begin{array}{l}\text { DL } \\
(\%)\end{array}$ \\
\hline CS-SA & $22 \pm 0.98$ & $0.247 \pm 0.025$ & $36.4 \pm 0.71$ & & \\
CS-SA/DOX & $55.4 \pm 4.95$ & $0.298 \pm 0.037$ & $36.5 \pm 1.1$ & 81.23 & 10.65 \\
\hline
\end{tabular}

Note: Data represent the mean \pm standard deviation $(n=3)$.

Abbreviations: CS-SA/DOX, doxorubicin-loaded stearic acid-grafted chitosan; $d_{n}$, number average diameter; PI, polydispersity index of the diameter; $\zeta$, zeta potential; $\mathrm{EE}$, drug entrapment efficiency; $\mathrm{DL}$, drug loading content. 

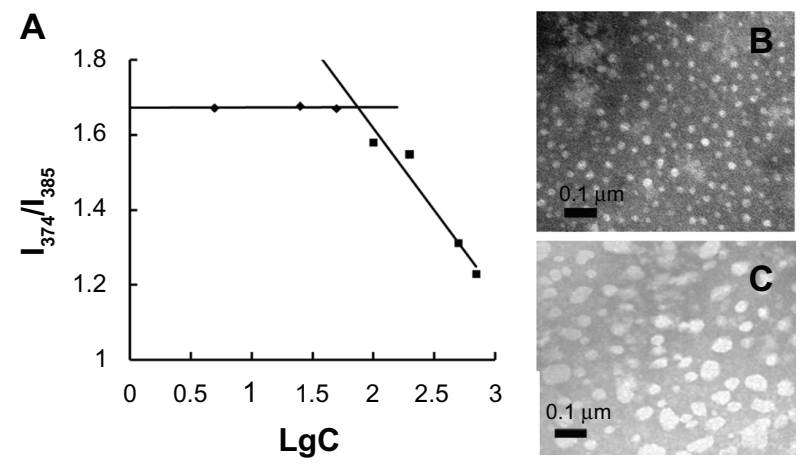

Figure 2 Fluorescence intensity ratio of $I_{1} / I_{3}$ plotted against logarithm of stearic acid-grafted chitosan (CS-SA) concentration (A); transmission electron microscopy images of CS-SA micelles (B); doxorubicin loaded CS-SA micelles (C).

intensity inside cells increased gradually and significant fluorescence was observed after 6 hours' incubation. The CS-SA micelles displayed an excellent ability to transport across cell membrane. It was assumed that endocytosis

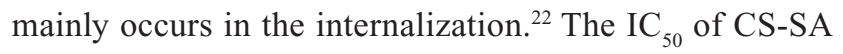
against bEnd. 3 was $237.6 \pm 6.61 \mu \mathrm{g} / \mathrm{mL}$, determined by MTT assay.
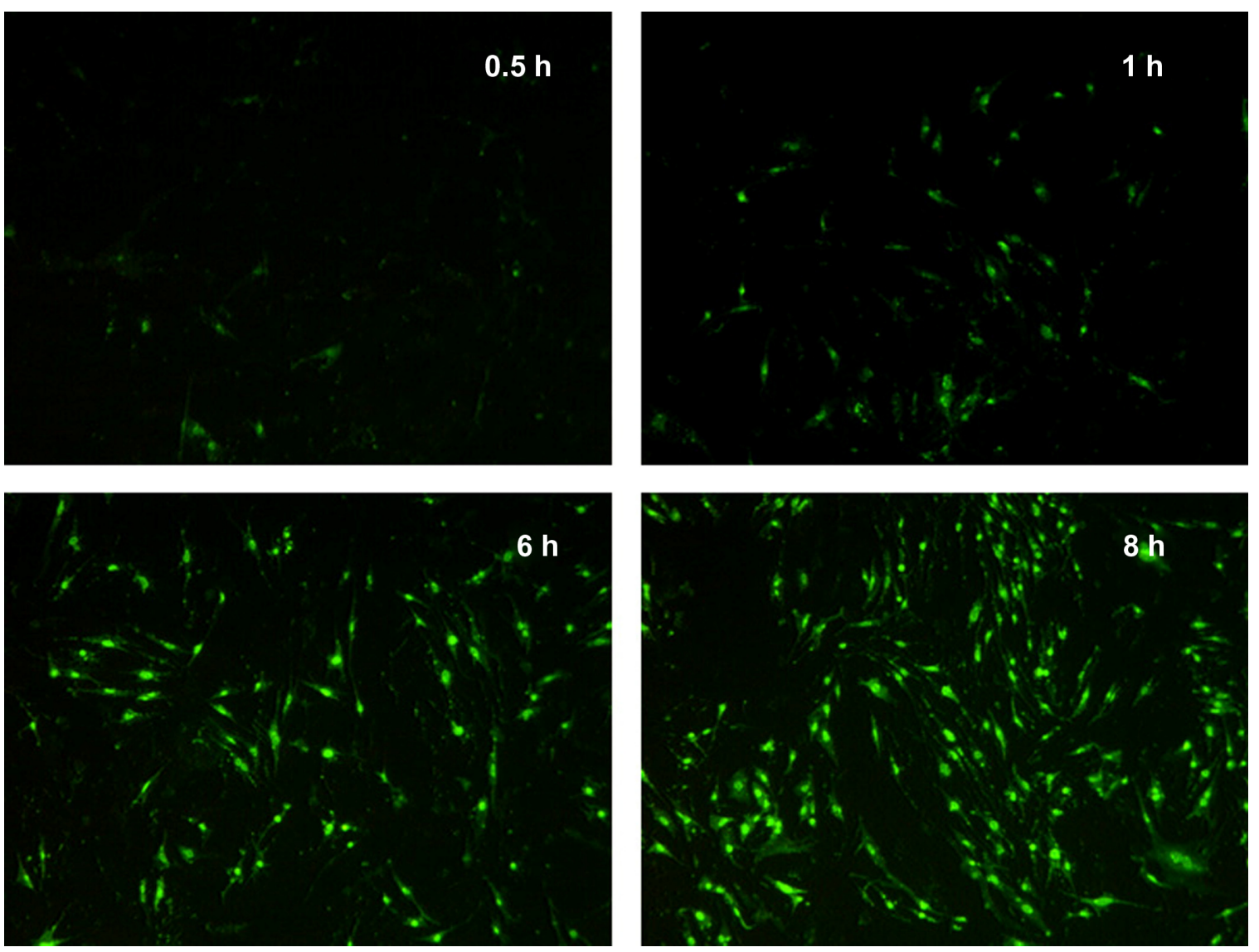

Figure 3 Fluorescence images of bEnd.3 after incubation with fluorescein isothiocyanate-labeled stearic acid-grafted chitosan micelles for 0.5 , I, 6 , and 8 hours. 


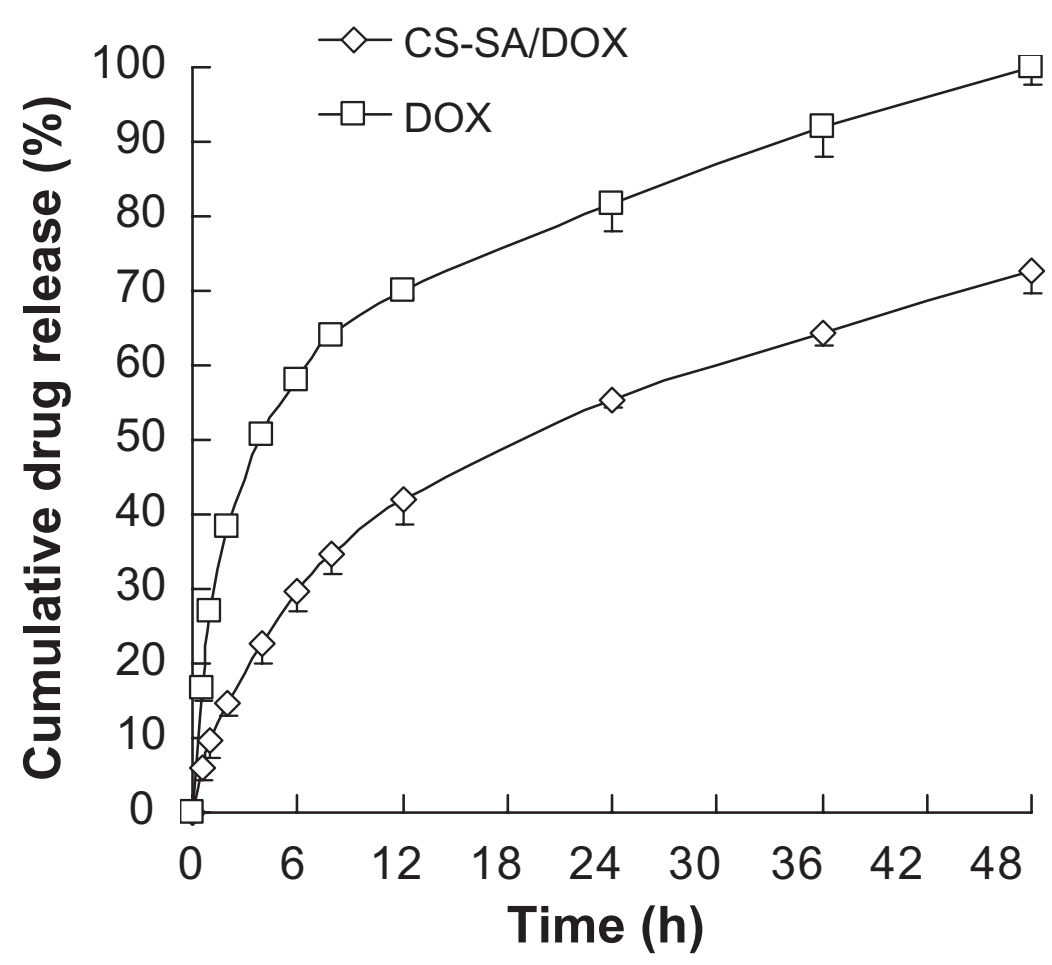

Figure 4 In vitro release profiles of doxorubicin (DOX)-loaded stearic acid-grafted chitosan (CS-SA) micelles in phosphate-buffered saline (pH 7.2).

CRI Maestro in vivo imaging system. Images were taken at different time points within 30 minutes after injection. As shown in Figure 5, CS-SA/DiR was strongly accumulated in brain 5 minutes after injection. The fluorescence gradually decreased and was still detectable 30 minutes after injection by observing ex vivo brain samples. Taking into account that fluorescence was not seen in brain treated with free DiR (not shown), the fast distribution of DiR in brain could be mainly ascribed to the permeability of CS-SA across BBB.

\section{In vivo distribution study}

In vivo distribution study of CS-SA/DOX micelles was performed in comparison with free DOX $\cdot \mathrm{HCl}$. The HPLC analysis system for in vivo DOX detection was established. Baseline separation of DOX was achieved. Recoveries for different tissues were all above $60 \%$ (data not shown), which could be acceptable in the analysis of DOX in vivo. ${ }^{26}$

The DOX level in brain was detected at different time points, as presented in Figure 6. It was found that DOX was able to distribute rapidly throughout brain, with the highest drug level of $1.01 \% / \mathrm{g}$ achieved at 15 minutes after injection. Then drug level gradually decreased. It maintained above $0.45 \% / \mathrm{g}$ for 1 hour and did not arrive at $0.2 \% / \mathrm{g}$ until 2 hours after administration.

The plasma concentration versus time profiles of CS-SA/ DOX micelles and free $\mathrm{DOX} \cdot \mathrm{HCl}$ is shown in Figure 7.
$5 \mathrm{~min}$

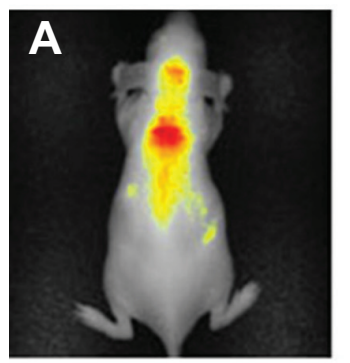

$10 \min$

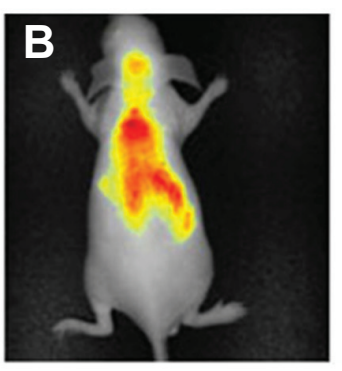

$15 \min$

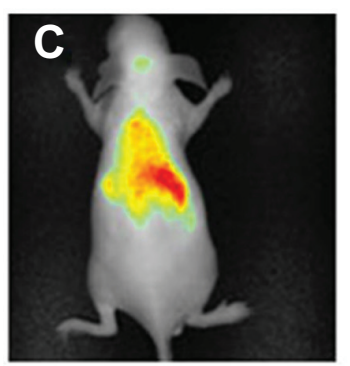

$30 \mathrm{~min}$

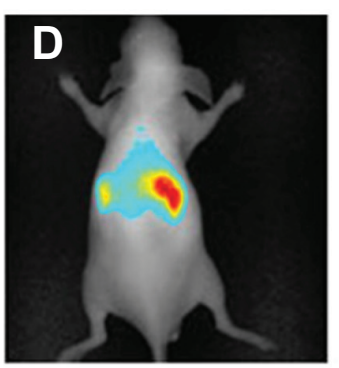

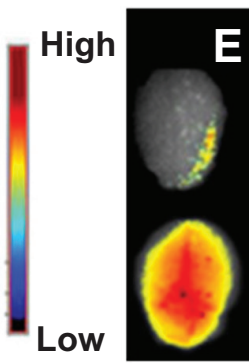

Figure 5 (A-E) In vivo images of stearic acid-grafted chitosan/DiR. Images A-D were taken within 30 minutes after injection; $\mathbf{E}$ is ex vivo brain at 30 minutes after injection, compared with blank brain. 


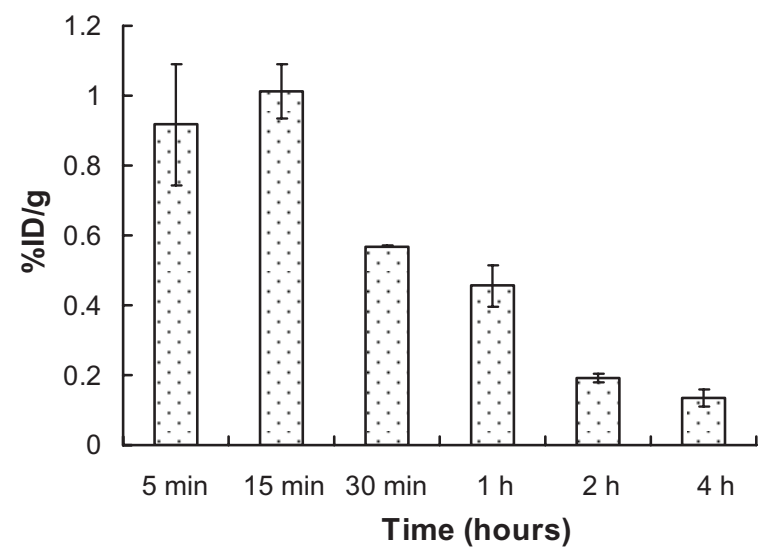

Figure 6 Brain distribution after injection of doxorubicin-loaded stearic acid-grafted chitosan at a dose of $2.5 \mathrm{mg} / \mathrm{kg}$ (equivalent of doxorubicin).

Note: Data represent the mean \pm standard deviation. $\mathrm{n}=3$.

According to analysis, the area under the curve was increased 1.97 -fold by using CS-SA/DOX micelles, compared to $\mathrm{DOX} \cdot \mathrm{HCl}$. The pharmacokinetics parameters were obtained by DAS 2.0 analysis and listed in Table 2 in detail. Biodistribution of CS-SA/DOX in other tissues was also investigated. Figure 8 illustrates the distribution of DOX at 0.5 and 2 hours following administration. It was found that drug was mainly located in lung, liver, and spleen. It is worthy of note that the amount of DOX in heart was reduced by half by CS-SA/DOX, compared with DOX $\cdot \mathrm{HCl}$.

\section{Discussion}

In vitro uptake study showed that CS-SA displayed excellent internalization ability on bEnd.3. The result was consistent with previous studies, in which CS-SA exhibited excellent

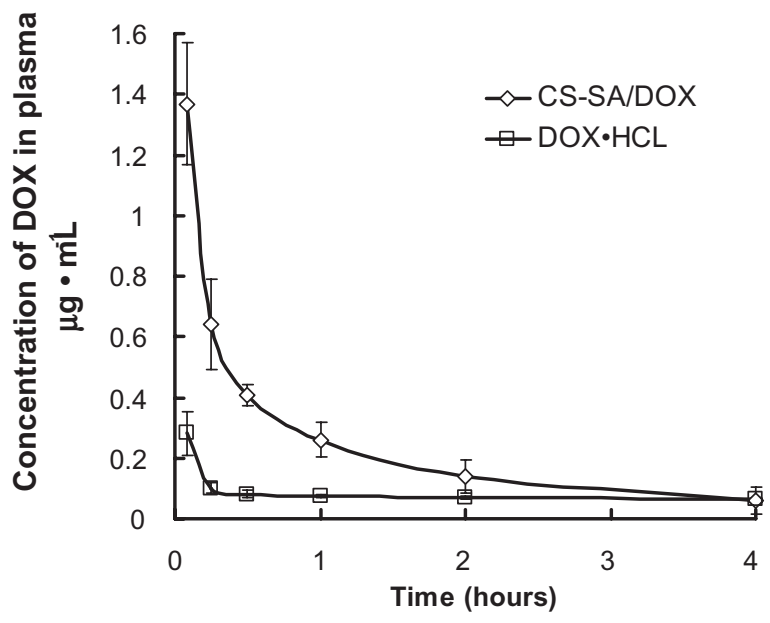

Figure 7 Plasma concentration versus time profiles of doxorubicin (DOX) following intravenous injection of DOX-loaded stearic acid-grafted chitosan (CS-SA/DOX) and DOX-hydrochloride (DOX HCL), both at a dose of I mg/kg (equivalent of DOX).

Note: Data represent the mean \pm standard deviation. $n=5$.
Table 2 Pharmacokinetic parameters in rat after intravenous administration of CS-SA/DOX and DOX. $\mathrm{HCl}$

\begin{tabular}{lll}
\hline Kinetic parameter & DOX $\cdot \mathbf{H C l}$ & CS-SA/DOX \\
\hline AUC $(0-\mathrm{t})$ & $0.326 \pm 0.025$ & $0.969 \pm 0.108$ \\
$\mathrm{MRT}(0-\mathrm{t})$ & $1.705 \pm 0.072$ & $0.909 \pm 0.174$ \\
$\mathrm{CLz}$ & $0.656 \pm 0.409$ & $1.005 \pm 0.13$ \\
$\mathrm{Vz}$ & $12.59 \pm 0.971$ & $1.266 \pm 0.246$ \\
$\mathrm{C}_{\max }$ & $0.293 \pm 0.074$ & $1.344 \pm 0.214$ \\
\hline
\end{tabular}

Abbreviations: CS-SA/DOX, doxorubicin-loaded stearic acid-grafted chitosan; $\mathrm{HCl}$, hydrochloride; AUC, area under curve; MRT, mean retention time.

cellular uptake on other tumor cells as well. ${ }^{19,22}$ In vitro toxicity study indicated that CS-SA could be used as a relatively safe carrier for brain-targeting drug delivery.

The rapid distribution of DOX in brain by quantification study agreed well with the in vivo imaging results. Meanwhile, no drug signal was detected in brain for the $\mathrm{DOX} \cdot \mathrm{HCl}$ group. Therefore, high drug levels in brain could be virtually attributed to BBB transport ability exerted by CS-SA micelles. The large amount of DOX accumulated in brain demonstrated the superior advantage of CS-SA micelles for brain targeting in contrast with the other kinds of nanoparticles whose average drug level in brain was merely $0.1 \% / \mathrm{g}-0.2 \% / \mathrm{g} .{ }^{27-31}$

Transport across BBB for micelles might be related to the high lipophicity increased by SA with a substitute degree of $26 \%$. Moreover, another important contribution to the successful transport of DOX into brain could be bypassing the P-gp effect. The efflux system (eg, P-gp), highly expressed on endothelial cells, effectively pumps exogenous agents from brain back to blood. ${ }^{2}$ Being the substrate of P-gp prevented the entry of DOX to brain. It was assumed that CS-SA/DOX micelles enhanced distribution of incorporated DOX in brain by masking physicochemical properties of DOX and thus bypassing P-gp recognition. However, it was noted that a dramatic decrease of DOX in brain was observed. The specific mechanism remained unknown and requires further investigation.

The large amount of DOX in lung might be associated with the particle size. The uptake of micelles by macrophages in the reticuloendothelial system might explain the drug distribution in the liver and the spleen. Cardiac toxicity was one major concern in clinical application of adriamycin. CS-SA/DOX greatly lowered drug distribution in heart, thus reducing cardiac toxicity.

\section{Conclusion}

The synthesized amphipathic polymer (CS-SA) was able to self-assemble into micelles. These CS-SA micelles exhibited excellent internalization capability and relatively low 

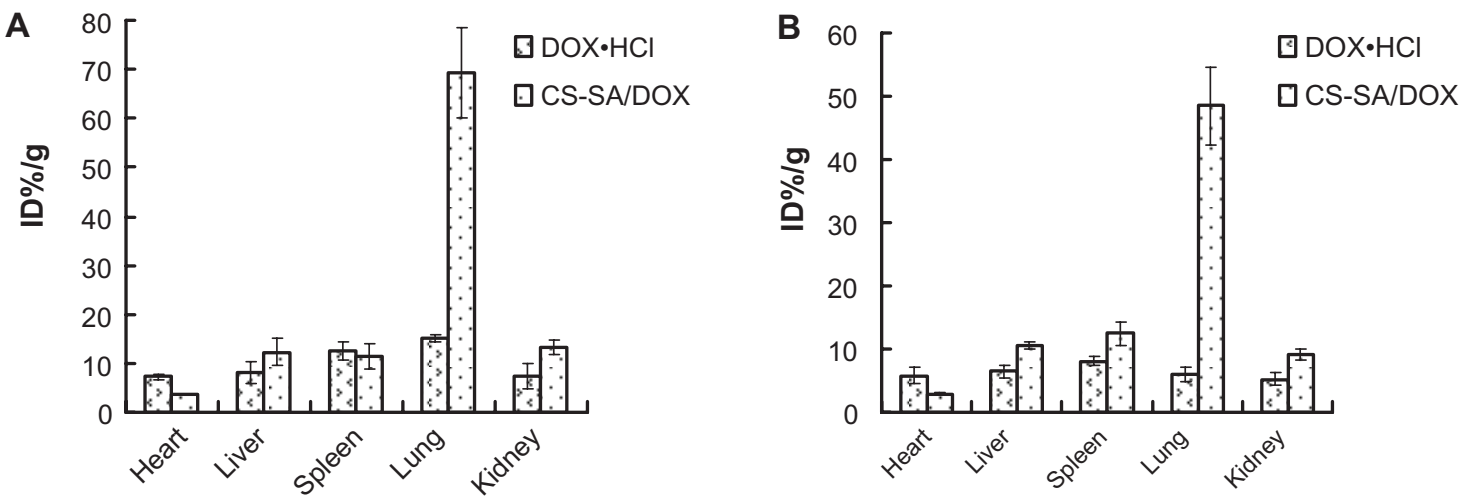

Figure 8 Tissue distribution at 0.5 and 2 hours after intravenous injection of doxorubicin-loaded stearic acid-grafted chitosan (CS-SA/DOX) and DOX-hydrochloride $(\mathrm{DOX} \cdot \mathrm{HCl})$.

Note: Data represent the mean \pm standard deviation. $\mathrm{n}=3$.

cytotoxicity on bEnd.3. In vivo imaging displayed a rapid distribution of CS-SA in brain. DOX was entrapped into CS-SA micelles with a high drug-loading capacity, and the DOX-loaded micelles exhibited slow release behavior in vitro. CS-SA/DOX micelles showed a therapeutic effect on C6 in vitro. A relatively high amount of DOX was found in brain, while a lower amount of drug was accumulated in heart. A longer retention time in brain would be achieved in treatment of brain tumor through the EPR effect of a nanosized micellar delivery system. It was confirmed that CS-SA possessed unique ability to transport across BBB both in vitro and in vivo. In conclusion, CS-SA could be exploited as a potential carrier to effectively deliver drugs across BBB and into brain.

\section{Acknowledgments}

We appreciate the financial support from the National Basic Research Program of China (973 Program) under Contract 2009CB930300, Key Project of Science and Technology of Zhejiang Province (grant no 2009C13037) and Zhejiang Provincial Program for the Cultivation of High-Level Innovative Health Talents.

\section{Disclosure}

The authors declare no conflicts of interest in this work.

\section{References}

1. Begley DJ. Delivery of therapeutic agents to the central nervous system: the problems and the possibilities. Pharmacol Ther. 2004; 104(1):29-45.

2. Kusuhara H, Sugiyama Y. Efflux transport systems for drugs at the blood-brain barrier and blood-cerebrospinal fluid barrier (Part 1). Drug Discov Today. 2001;6(3):150-156.

3. Batrakova EV, Kabanov AV. Pluronic block copolymers: evolution of drug delivery concept from inert nanocarriers to biological response modifiers. J Control Release. 2008;130(2):98-106.
4. Olivier JC. Drug transport to brain with targeted nanoparticles NeuroRx. 2005;2(1):108-119.

5. Gref R, Couvreur P, Barratt G, et al. Surface-engineered nanoparticles for multiple ligand coupling. Biomaterials. 2003;24(24): 4529-4537.

6. Calvo P, Remunan-Lopez C, Vila-Jato JL, et al. Development of positively charged colloidal drug carriers: chitosan-coated polyester nanocapsules and submicron-emulsions. Colloid Polym Sci. 1997;275(1):46-53.

7. Couvreur P, Vauthier C. Polyalkylcyanoacrylate nanoparticles as drug carrier: present state and perspectives. J Control Release. 1991;17(2):187-198.

8. Gulyaev AE, Gelperina SE, Skidan IN, et al. Significant transport of doxorubicin into the brain with polysorbate 80 -coated nanoparticles. Pharm Res. 1999;16(10):1564-1569.

9. Steiniger SCJ, Kreuter J, Khalansky AS, et al. Chemotherapy of glioblastoma in rats using doxorubicin loaded nanoparticles. Int $J$ Cancer. 2004;109(5):759-767.

10. Tosi G, Costantino L, Rivasi F, et al. Targeting the central nervous system: in vivo experiments with peptide-derivatized nanoparticles loaded with Loperamide and Rhodamine-123. J Control Release. 2007;122(1):1-9.

11. Alyautdin R, Gothier D, Petrov V, et al. Analgesic activity of the hexapeptide dalargin adsorbed on the surface of polysorbate 80-coated poly (butyl cyanoacrylate) nanoparticles. Eur J Pharm Biopharm. 1995;41(1):44-48.

12. Kreuter J, Alyautdin RN, Kharkevich DA, et al. Passage of peptides through the blood-brain barrier with colloidal polymer particles (nanoparticles). Brain Res. 1995;674(1):171-174.

13. Kusuhara H, Suzuki H, Sugiyama Y. The role of P Glycoprotein and canalicular multispecific organic anion transporter in the hepatobiliary excretion of drugs. J Pharm Sci. 1998;87(9):1025-1040.

14. Loe DW, Deeley RG, Cole SP. Biology of the multidrug resistanceassociated protein, MRP. Eur J Cancer. 1996;32(6):945.

15. Hipfner DR, Deeley RG, Cole SPC. Structural, mechanistic and clinical aspects of MRP1. Biochim Biophys Acta. 1999;1461(2):359-376.

16. Park JH, Saravanakumar G, Kim K, et al. Targeted delivery of low molecular drugs using chitosan and its derivatives. Adv Drug Deliver Rev. 2010;62(1):28-41.

17. Mistry A, Stolnik S, Illum L. Nanoparticles for direct nose-to-brain delivery of drugs. Int J Pharm. 2009;379(1):146-157.

18. Hu FQ, Zhao MD, Yuan H, et al. A novel chitosan oligosaccharide-stearic acid micelles for gene delivery: properties and in vitro transfection studies. Int J Pharm. 2006;315(1-2):158-166.

19. Hu FQ, Liu LN, Du YZ, et al. Synthesis and antitumor activity of doxorubicin conjugated stearic acid-g-chitosan oligosaccharide polymeric micelles. Biomaterials. 2009;30(36):6955-6963. 
20. Hu F Q, Wu X, Du YZ, et al. Cellular uptake and cytotoxicity of shell crosslinked stearic acid-grafted chitosan oligosaccharide micelles encapsulating doxorubicin. Eur J Pharm Biopharm. 2008;69(1): 117-125.

21. Hu FQ, Ren GF, Yuan H, et al. Shell cross-linked stearic acid grafted chitosan oligosaccharide self-aggregated micelles for controlled release of paclitaxel. Colloids Surf B Biointerfaces. 2006;50(2):97-103.

22. Yuan H, Lu L, Du Y Z, et al. Stearic acid-g-chitosan polymeric micelle for oral drug delivery: in vitro transport and in vivo absorption. Mol Pharm. 2011;8(1):225-238.

23. Huang M, Ma Z, Khor E, et al. Uptake of FITC-chitosan nanoparticles by A549 cells. Pharm Res. 2002;19(10):1488-1494.

24. Kohori F, Yokoyama M, Sakai K, et al. Process design for efficient and controlled drug incorporation into polymeric micelle carrier systems. J Control Release. 2002;78(1-3):155-163.

25. Yu JM, Li YJ, Qiu LY, et al. Polymeric nanoparticles of cholesterol modified glycol chitosan for doxorubicin delivery: preparation and in vitro and in vivo characterization. J Pharm Pharmacol. 2009;61(6):713-719.

26. Stefanie W, Alexander K, Svetlana G, et al. Kinetics of transport of doxorubicin bound to nanoparticles across the blood-brain barrier. J Control Release. 2011;154(1):103-107.
27. Lode J, Fichtner I, Kreuter J, et al. Influence of surfacemodifying surfactants on the pharmacokinetic behavior of 14C-poly (methylmethacrylate) nanoparticles in experimental tumor models. Pharm Res. 2001;18(11):1613-1619.

28. Ambruosi A, Khalansky AS, Yamamoto H, et al. Biodistribution of polysorbate 80-coated doxorubicin-loaded [14C]-poly(butyl cyanoacrylate) nanoparticles after intravenous administration to glioblastoma-bearing rats. J Drug Target. 2006;14(2):97-105.

29. Brigger I, Morizet J, Laudani L, et al. Negative preclinical results with stealth nanospheres-encapsulated doxorubicin in an orthotopic murine brain tumor model. J Control Release. 2004;100(1):29-40.

30. Brigger I, Morizet J, Aubert G, et al. Poly(ethylene glycol)-coated hexadecylcyanoacrylate nanospheres display a combined effect for brain tumor targeting. J Pharmacol Exp Ther. 2002;303(3):928-936.

31. Calvo P, Gouritin B, Chacun H, et al. Long-circulating PEGylated polycyanoacrylate nanoparticles as new drug carrier for brain delivery. Pharm Res. 2001;18(8):1157-1166.
International Journal of Nanomedicine

\section{Publish your work in this journal}

The International Journal of Nanomedicine is an international, peerreviewed journal focusing on the application of nanotechnology in diagnostics, therapeutics, and drug delivery systems throughout the biomedical field. This journal is indexed on PubMed Central, MedLine, CAS, SciSearch $\AA$, Current Contents ${ }^{\circledR} /$ Clinical Medicine,

\section{Dovepress}

Journal Citation Reports/Science Edition, EMBase, Scopus and the Elsevier Bibliographic databases. The manuscript management system is completely online and includes a very quick and fair peer-review system, which is all easy to use. Visit http://www.dovepress.com/ testimonials.php to read real quotes from published authors. 